\title{
Ergonomic considerations in natural orifice translumenal endoscopic surgery (NOTES): A case study
}

\author{
Cristina Tessier, Likun Zhang, and Caroline G.L. Cao* \\ Department of Mechanical Engineering, Tufts University, 200 College Avenue, Medford, MA 02155 United States
}

\begin{abstract}
Surgical technique evolved from traditional open surgery to laparoscopic surgery, offering patients reduced pain and quick recovery. Recently, a new approach called natural orifice translumenal endoscopic surgery (NOTES) has emerged. In NOTES, procedures may be transvaginal, transgastric, or transrectal or transesophageal. NOTES procedures present new ergonomic challenges to surgeons due to visualization and tissue manipulation challenges. An ergonomic analysis of NOTES cholecystectomy was conducted. As there is currently no standardization for performing NOTES, this analysis presents the results as a case study. Results showed NOTES procedures impose unique limitations on the surgeon's ability to perform surgical tasks autonomously, requiring collaboration and coordination amongst the surgical team members.
\end{abstract}

Keywords: natural orifice translumenal endoscopic surgery (NOTES); laparoscopic surgery; task analysis; ergonomics

\section{Introduction}

The first published human case of a diagnostic laparoscopic procedure was performed in the early 1950s [15]. The first human in-vivo laparoscopic cholecystectomy was performed in 1985 in Germany[13]. Since then, laparoscopy has become the preferred method for several surgical procedures, including gallbladder removal or cholecystectomy. The technique and tools have been refined to bring it to its current state as the preferred and most common method of gallbladder removal [1]. There has been a movement in the intervening years towards even less invasive surgical techniques, such as single incision laparoscopic surgery, mini-laparoscopic surgery, and natural orifice translumenal endoscopic surgery (NOTES) [2]. The 2004 Kalloo case using a porcine model is widely credited as the first proof-of-concept demonstration for NOTES [11]. While there are variations on what may be considered a NOTES procedure, the definition for the purposes of this research is, a surgical procedure which traverses the lumen of an organ (e.g., colon, oesophagus, etc.) through a natural orifice in the human body (e.g., the anus, the mouth, etc.) with a visualization tool (e.g., laparoscope or endoscope) and various instruments for the facilitation of the surgery. The common approaches used in human cases since January 1, 2004 have been transvaginal, transgastric, transrectal, and transesophageal NOTES [2].

The most common human NOTES case reported since that time is the transvaginal cholecystectomy (Fig 1) with the use of at least one other port of entry in the abdominal wall, usually at the umbilicus [2]. For this reason, the analysis presented here is specific to cholecystectomy performed with the single-port assisted NOTES.

\footnotetext{
* Corresponding author. E-mail: caroline.cao@tufts.edu. Tel: 617-627-2484 Fax: 617-627-3058
} 


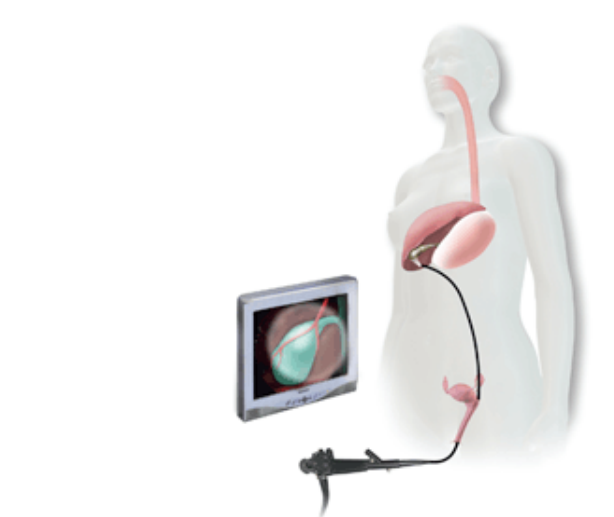

Fig 1. Transvaginal cholecystectomy. Photo courtesy of Dr. Mark Bessler, MD.

\section{Problem}

Minimally invasive surgery presents ergonomic challenges to clinicians that differ from the challenges seen with open techniques. For example, tools used for laparoscopic procedures have working ends on long rigid shafts. The degrees of freedom for manipulation are reduced from 6 in open surgery to 4 in laparoscopic surgery, which forces the surgeon's arms and wrists to end up in odd, unnatural positions. This is not only uncomfortable, but leads to muscle fatigue [5]. Haptic feedback (i.e., the sense of touch) is reduced, largely due to the indirect manipulation with laparoscopic instruments [16]. The surgeon's hand-eye coordination is severely disrupted in laparoscopic surgery, due to the indirect view of the operative field [6]. Other challenges in laparoscopic surgery include the fulcrum effect [3] (which results in a mirrored image between tool handle movement direction and the corresponding tool end-effector movement), lack of depth perception on the $2 \mathrm{D}$ display, and varying image magnification on the monitor.

NOTES procedures appear to suffer from many of the same problems as in laparoscopic surgery, as well as several ergonomic issues that are unique to NOTES. An analysis was performed based on case observations in the operating room of NOTES cholecystectomy procedures.

\section{Cholecystectomy}

\subsection{Laparoscopic cholecystectomy}

For a typical laparoscopic cholecystectomy procedure, the patient is placed under general anaethesia and an incision is made either trans-umbilically or sub-umbilically through the peritoneum. A port is placed through the incision for insufflation with $\mathrm{CO}_{2}$ and insertion of laparoscope. At least two other ports are similarly placed for the insertion of tools as shown in Fig 2.

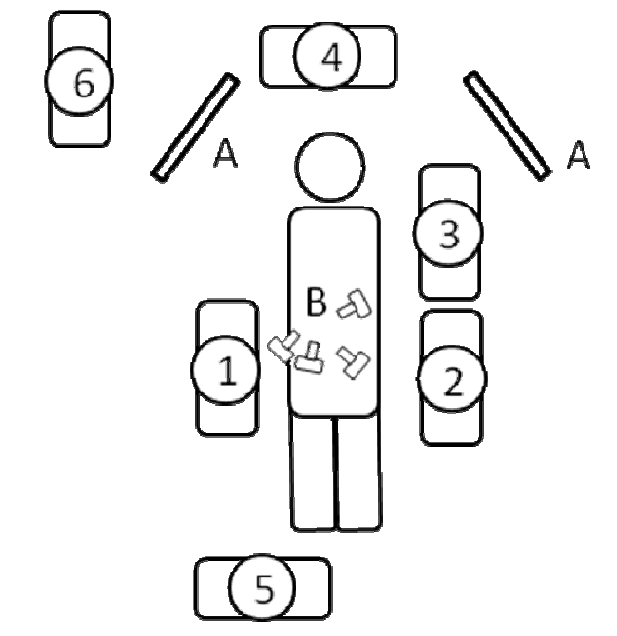

Fig 2. Operating room layout for a typical laparoscopic cholecystectomy. 1=Assisting surgeon; 2=Camera holder/assisting surgeon; 3=Attending surgeon; 4=Anaesthesiologist; 5=Scrub nurse; 6=Circulating nurse; $A=$ Monitor displaying laparoscope image.

While the gallbladder is pulled up and in tension, usually by an assisting surgeon, the attending surgeon dissects the tissue surrounding the common hepatic duct, cystic duct, and the cystic artery. Clips are placed on the cystic artery and duct and artery is cut between the clips. The gall bladder is dissected from the liver bed and removed with a special bag. An inspection of the site is performed to verify there is no fluid leakage. The area is washed if necessary. The abdomen is desufflated and the ports closed. The incisions are dressed and the patient is awakened [12].

\subsubsection{Ergonomic evaluations}

The physical and psychological ergonomic issues involved in laparoscopic procedures such as clinician position, port placement, tool angle, monitor placement, and mental workload have been noted and studied extensively [4-6, 9].

In the early days of laparoscopy, a detailed analysis of several laparoscopic surgeries was conducted 
by Cao [7]. This work deconstructed the procedures into steps, then into the tasks within those steps, the subtasks within those tasks, and the motions within those subtasks. The motions involved with the use of the typical tool set were also analyzed. Results showed that the basic surgical tasks in laparoscopic surgery required deliberate movement of the tools with limited degrees of freedom. Indeed, translation and rotation of the tool were performed separately [7] The poor design of laparoscopic tools and limited degrees of freedom for manipulation make the difficult tasks such as suturing and tying knots even more difficult to learn and master than in open surgery.

Youseff et. al. varied the standing positions and hand usage of subjects performing simulated laparoscopic cholecystectomy in order to evaluate the physical ergonomics and mental workload involved in each of the variations [19]. They found that standing in the position between the patient's legs provides better ergonomics than standing to one side of the patient. A NASA-TLX assessment of both techniques showed that there was a significantly higher amount of physical demand, effort, and frustration perceived with the side standing technique vs. the between-legs technique. Van Det et. al. compared neck posture in a group of 16 surgical team members when they performed a laparoscopic cholecystectomy in a conventional operating room vs. a suite with monitors configured specifically for minimally invasive procedures [18]. The results showed that the suspended and adjustable monitors in the modern MIS suite can improve ergonomic quality of the neck posture for the operating team, as they allow the surgical team to stand straight in front of monitors with minimal neck rotation. Talebpour and Panahi examined an ergonomic configuration of trocar port placement for laparoscopic cholecystectomy (i.e., a 120-degree angle between the two trocars with the laparoscopic tools, with the center in the umbilicus; a $7-10 \mathrm{~cm}$ distance between the endoscope trocar and the working trocars; and at least a $15-20 \mathrm{~cm}$ distance between the endoscope port and the gallbladder) [17]. They suggested that ergonomic configuration of the trocar ports can provide the surgeon with greater ease in performing surgical procedures, with a better exposure of the field and the gallbladder.

During laparoscopic surgery, the surgeon's handeye coordination is often disrupted by the incongruent mapping between the orientation of the endoscopic view and the actual operative field. Studies have shown that best performance is achieved when the endoscopic image is perfectly aligned with the actual task space; performance degrades as the alignment deviates from 0 -degree, and the degradation increases as the angle of deviation increases $[6,8,20]$. It is important to align the endoscopic image with the actual surgical filed to maintain visuomotor congruency for the surgeon in laparoscopic surgery.

Those studies shed light on the design of laparoscopic tools and procedural guidelines to optimize physical ergonomics and decrease mental workload.

\subsection{NOTES cholecystectomy}

\subsubsection{Origins}

The first series of human transvaginal cholecystectomy cases was performed by a research group led by Dr. Zorron in March 2007 [21]. Since then, there have been over 300 reported human cases of transvaginal cholecystectomy [2].

\subsubsection{Overview}

In a typical NOTES cholecystectomy procedure, the patient is placed under general anaesthesia. For hybrid procedures where instruments are also introduced transabdominally, an incision is made through the peritoneum at the umbilicus for the placement of a port as shown in Fig 3. This is performed in most cases and may be done in part to provide direct visualization of the entrance of tools transvaginally into the abdomen. 


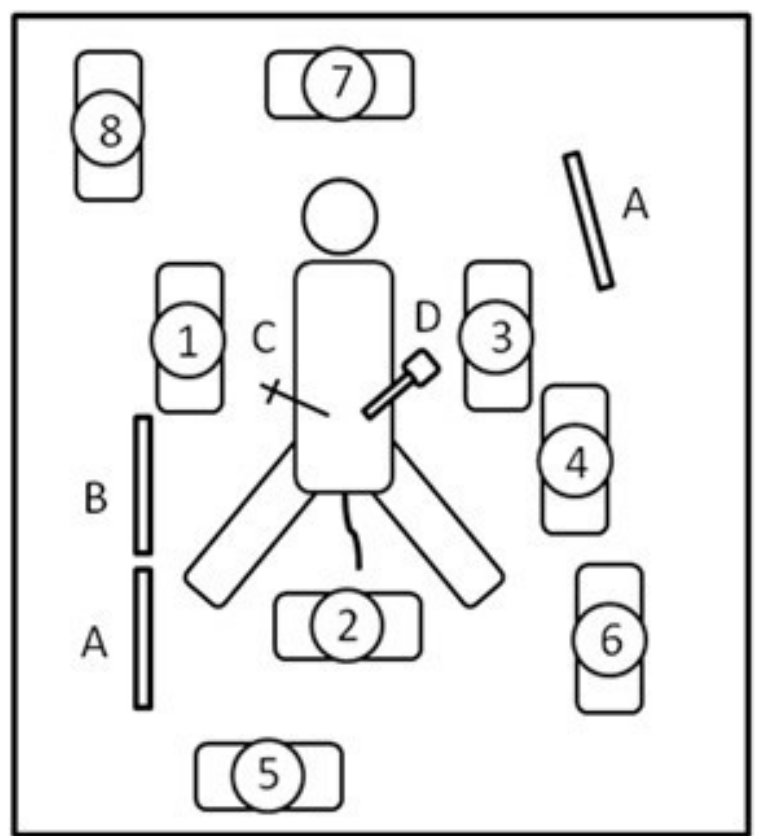

Fig 3. Typical layout in the operating room for a transvaginal NOTES cholecystectomy. $1=$ surgeon holding mini-laparoscopic grasper; $2=$ gastroenterologist holding endoscope; $3=$ surgeon holding laparoscope; 4=assisting surgeon; 5=endoscopy technician; $6=$ scrub nurse; $7=$ anaesthesiologist; $8=$ circulating nurse; $\mathrm{A}=$ monitor displaying endoscopic image; $\mathrm{B}=$ monitor displaying laparoscopic image; $\mathrm{C}=$ mini-laparoscopic grasper; $\mathrm{D}=$ laparoscope

The abdomen is insufflated through the laparoscope port. The cervix is incised and the mucosa cauterized. The peritoneum is cut to enter the abdominal cavity. The endoscope and tools are introduced through the vaginal port. A mini-laparoscopic grasper or other needle-based tool is placed in the abdomen to grasp and hold taut the gallbladder. Tools such as graspers and manipulators are used for dissection of the common hepatic duct, cystic duct, and the cystic artery. The remaining steps are similar to those outlined in Section 3.1, with the gallbladder exiting the abdomen transvaginally [14].

\subsubsection{Ergonomic evaluation}

To-date, there has been no systematic analysis of the ergonomics of NOTES and its implications for instrumentation design or skills training. One of the few existing studies conducted focused on the neuroergonomics of NOTES [10]. The brain behavior of experts and novices (divided based on endoscopy experience) during a NOTES spatial navigation task was examined. Despite the significant difference in the location of prefrontal cortical activity, both groups seemed to rely on visuospatial working mem- ory to maintain a representation of the desired course during the experiment. The findings suggested a necessity to design and integrate navigational aids to facilitate the NOTES procedure, and enhance performance for surgeons.

\section{Method}

4.1. A transvaginal NOTES cholecystectomy was observed at a local hospital in the Boston area. The surgical team members' positions relative to each other, the patient, the monitors, and the tools were noted. These elements are illustrated in Fig 3. Handwritten notes were taken throughout the procedure, with particular attention paid to tool use. Surgical team members occasionally highlighted elements of the case for the researchers' benefit. A summary and analysis of key differences between the observed NOTES case and a typical laparoscopic cholecystectomy are presented here.

\section{Results and Analysis}

\section{1. "Coupling"}

In the observed NOTES case, the camera displaying the primary image was at the end of the same flexible body that housed the two tools used for dissection, cautery, etc. These tools were continually being advanced, retracted, and rotated. This movement could be seen creating reaction forces on the endoscope. The tools also experienced reaction forces from the tissues with which they made contact. These forces were observed to transfer easily to the endoscope and therefore jostle the image being displayed for the gastroenterologist or surgeon. To a certain degree, the camera and tools were "coupled." In other words, movement of the hand in controlling the surgical tools also resulted in similar movement of the endoscopic image, which was sometimes amplified. This image movement increased the difficulty of accomplishing precise and accurate tool use. Compared to laparoscopic surgery, where the tool ports and scope ports are separate and therefore independently of each other, the coupling of the tool and camera motion is an example of an ergonomic issue unique to NOTES. 


\subsection{Flexible scope manipulation}

The unique navigation and articulation skills required for flexible endoscope use may present difficulty to a surgeon accustomed to rigid laparoscopes. With rigid scopes, the surgeon can advance/retract, rotate, or change the angle of the camera about the fulcrum of the port. With a flexible scope, on the other hand, the surgeon can also bend the tip of the scope in two planes by rotating dials on its proximal end. Depending on how far into the anatomy the scope is advanced, the surgeon cannot rely on the same "pushability" (i.e., force transfer between proximal and distal ends) as with a rigid scope. The body of a flexible endoscope is also able to bend in reaction to the surrounding tissue when the dials are not locked. These endoscope skills are well developed in an expert gastroenterologist but not necessarily in a general surgeon.

In the observed case, both a general surgeon and a gastroenterologist were present, and took turns using the endoscope to perform the same tasks. It was observed that the gastroenterologist had better economy of motion, less awkward hand position, and more relaxed arm position than the general surgeon for these tasks. Fig 4 illustrates the arm position of the gastroenterologist for the majority of the procedure. The handle of the scope was positioned so that the dials faced up and the elbow could remain close to the side while the right hand turned the dials. The surgeon, however, turned the handle so that the dials faced to the right, creating a hand position that naturally lifted the elbow out from the body. The gastroenterologist also kept the proximal controls closer to the flexible end of the scope than the surgeon, for easier movement of the right hand back and forth between the dials to the scope as needed.

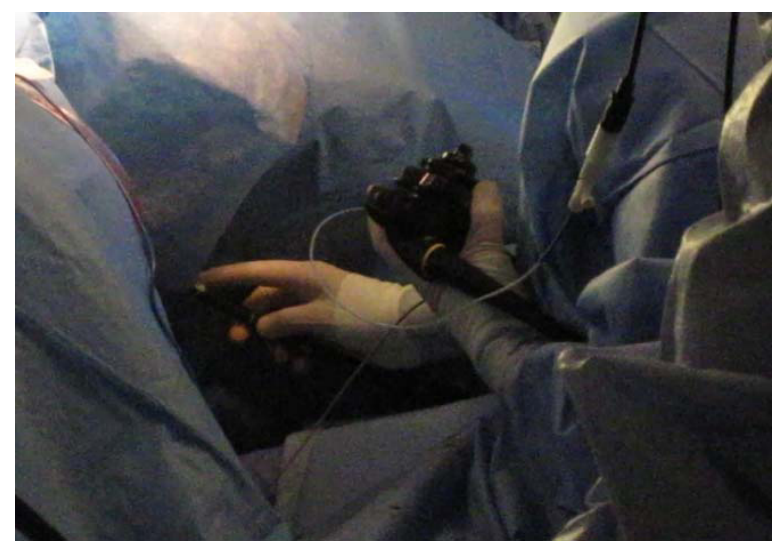

Fig 4. Hand and arm position of gastroenterologist.

\subsection{Division of labor for dissection}

Team collaboration is another unique feature of NOTES. The endoscope operator (i.e., the gastroenterologist for most of the case) was responsible for the rotation, advance/retract, and tip articulation of the endoscope. The endoscope operator was also responsible for the rotation and advance/retract of the flexible tools within the scope. The surgeon, standing to the patient's right was responsible for the open/close action of the dissector tool. Successful dissection required the smooth execution of the following sequence of actions:

- The endoscope operator turning a dial on the endoscope to aim the tool tip,

- The endoscope operator grasping and pushing the tool shaft to advance it towards the target tissue,

- The endoscope operator cueing the surgeon to close the dissector on the target tissue,

- The surgeon grasping the dissector handle to close the end-effector on the tissue,

- The endoscope operator pressing a foot pedal to initiate cautery.

In a conventional laparoscopic cholecystectomy, all of the actions involved in this dissection task are performed by one individual.

\subsection{Efficiency of tools}

In laparoscopy, a typical tool may be 5 or 10 millimeters in outer diameter when closed. With flexible endoscopy, a tool may be only a few millimeters in diameter. Accordingly, the length of the jaws of laparoscopic tools is longer than those used in NOTES. The result is smaller "bite sizes" during dissection, which, among other elements, contributes to longer procedure times in NOTES. The observed case, if performed laparoscopically, would have taken as little as 25 minutes. Actual procedure time from first incision was over 3 hours for the NOTES cholecystectomy.

\section{Conclusion}

From a technological perspective, there is a natural progression in surgical technique, evolving from traditional open surgery to minimally invasive laparoscopic surgery, to non-invasive surgery (i.e., natural orifice translumenal endoscopic surgery (NOTES)). Analyses revealed increased complexities of the 
NOTES technique compared with the laparoscopic technique. Results also showed that the human factors and ergonomics issues normally encountered in laparoscopic surgery (such as spatial disorientation and limited degrees of freedom in tool manipulation) are more pronounced in NOTES. Additional ergonomic issues are due to the fact that the surgical tools and instrumentation used for NOTES are borrowed from the laparoscopic tool box, which have not been developed to meet the increased task demands.

\section{Reference}

[1] A. Arezzo and M. Morino, Endoscopic surgery through single-port incision: time for a trial? Surg Endosc 25 (2011), 1709-1711.

[2] E. D. Auyang, B. F. Santos, D. H. Enter, E. S. Hungness, and N. J. Soper, Natural orifice translumenal endoscopic surgery (NOTES): a technical review, Arch Surg 144 (2009), 160-166.

[3] O. Ben-Porat, M. Shoham, and J. Meyer, Control design and task performance in endoscopic Teleoperation, Presence 9 (2000), 256-267.

[4] R. Berguer, Surgical technology and the ergonomics of laparoscopic instruments, Surg Endosc 12 (1997), 458-462.

[5] R. Berguer, D. L. Forkey, and W. D. Smith, Ergonomic problems associated with laparoscopic surgery, Surgical Endoscopy 13 (1999), 466-468.

[6] P. Breedveld and M. Wentink, Eye-hand coordination in laparoscopy - An overview of experiments and supporting Aids, Minimally Invasive Therapy and Allied Technologies 10 (2001), 155-162.

[7] C. Cao, C. L. MacKenzie, and S. Payandeh, S., Task and motion analyses in endoscopic surgery. Proceedings of the ASME Dynamic Systems and Controls Division. (Fifth Annual Symposium on Haptic Interfaces for Virtual Environment and Teleoperator Systems), DSC-Vol. 58 (1996), 583590.

[8] J. C. Gould and J. Frydman, Reverse-alignment surgical skills assessment, Surgical Endoscopy 21 (2007), 669-671.
[9] J. G. Holden, J. M. Flach, and Y. Donchin, Perceptualmotor coordination in an endoscopic surgery simulation, Surgical Endoscopy 13 (1999), 127-132.

[10] D. R. James, F. Orihuela-Espina, D. R. Leff, M. H. Sodergren, T. Athanasiou, A. W. Darzi, and G. Yang, The ergonomics of natural orifice translumenal endoscopic surgery (NOTES) navigation in terms of performance, stress, and cognitive behavior, Surgery 149 (2011), 525-533.

[11] A. N. Kalloo, V. K. Singh, S. B. Jagannath, H. Niiyama, S. L. Hill, C. A. Vaughn, C. A. Magee, and S. V. Kantsevoy, Flexible transgastric peritoneoscopy: a novel approach to diagnostic and therapeutic interventions in the peritoneal cavity, Gastrointest Endosc 60 (2004), 114-117.

[12] B. Navez. Laparoscopic cholecystectomy for acute cholecystitis, Epublication: WeBSurg.com, Feb 2001, 1(2). URL: http://www.websurg.com/ref/doi-ot02en012.htm

[13] W. Reynolds, The first laparoscopic cholecystectomy, JSLS 5 (2001), 89-94.

[14] J. Romanelli, Direct observation of transvaginal cholesytectomy case, July 29, 2011. Baystate Medical Center, Springfield, MA, U.S.

[15] G. Schlogel, Raoul Palmer and the coelio-surgical adventure from 1940 to 1995, Hist Sci Med 30 (1996), 281-287.

[16] H. G. Stassen, J. Dankelman, and K. A. Grimbergen, Manmachine aspects of minimally invasive surgery, Annual Reviews in Control 25 (2001), 111-122.

[17] M. Talebpour and M. Panahi, New aspects in laparoscopic cholecystectomy, J Laparoendosc Adv Surg Tech 17 (2007), 290-295.

[18] M. J. van Det, W. J. Meijerink, C. Hoff, M. A. van Veelen, and J. P. Pierie, Ergonomic assessment of neck posture in the minimally invasive surgery suite during laparoscopic cholecystectomy, Surg Endosc 22 (2008), 2421-2427.

[19] Y. Youssef, G. Lee, C. Godinez, E. Sutton, R. V. Klein, I. M. George, F. J. Seagull, and A. Park, Laparoscopic cholecystectomy poses physical injury risk to surgeons: analysis of hand technique and standing position, Surg Endosc 25 (2011), 2168-2174.

[20] L. Zhang and C. G. L. Cao, The effect of image orientation on a dynamic laparoscopic task, Human Factors and Ergonomics Society Annual Meeting 54 (2010), 774-778.

[21] R. Zorron, M. Filqueiras, L. C. Maggioni, L. Pombo, G. Lopes Carvalho, and A. Lacerda Oliveira, NOTES. Transvaginal cholecystectomy: report of the first case, Surg Innov 14 (2007), 279-283. 